\title{
Desempeño docente en los cursos complementa- rios de Inglés de la URACCAN-Recinto Universitario Nueva Guinea
}

\author{
Teaching performance in the Complementary English Courses at URACCAN, \\ Nueva Guinea Campus
}

\author{
Juan Carlos Ortiz Altamirano' \\ Rubby Idania Cruz Cano 2 \\ Juliana Francis Smith ${ }^{3}$
}

\section{Resumen}

Esta investigación ha evaluado el desempeño de los docentes en los cursos complementarios de Inglés de la Universidad de las Regiones Autónomas de la Costa Caribe Nicaragüense-Recinto Nueva Guinea, durante el primer semestre, 2018. El estudio se sustenta en una metodología con enfoque mixto, avalado por un diseño descriptivo y alcance exploratorio, por ello, se aplicaron instrumentos como guías para la revisión documental a materiales impresos y electrónicos, así como cuestionarios con una escala Likert a docentes y estudiantes de la universidad. Los principales hallazgos muestran la responsabilidad de los docentes en la planificación; la organización de los aprendizajes; el sistema de evaluación utilizado en los cursos; los procesos de comunicación y relaciones interpersonales; el trabajo en equipo y el nivel de motivación y su incidencia en la práctica de los docentes. Se concluye que las capacidades pedagógicas de los docentes son muy buenas, es decir, la mayoría brinda herramientas para que el estudiantado promueva su desarrollo personal y el de su comunidad, por medio de estrategias de motivación; sin embargo, no apuntan al desarrollo de metodologías pertinentes para facilitar el proceso educativo especialmente en las relaciones interculturales de género.

Palabras clave: Evaluación; desempeño; docencia; cursos complementarios; comunicación; motivación.

\section{Abstract}

This research has evaluated the teachers' performance in the Complementary English Courses of the University of the Autonomous Regions of the Nicaraguan Caribbean Coast-Nueva Guinea Campus, during the first semester, 2018. The study is based on a methodology with a mixed approach, endorsed by a descriptive design and exploratory scope, therefore, instruments were applied as guides for the documentary review of printed and electronic materials, as well as questionnaires with a Likert scale to university teachers and students. The main findings show the responsibility of teachers in lesson planning; the learning organization; the evaluation system used in the courses; communication processes and interpersonal relationships; team work; the motivation level and its incidence in the teachers practice. It is concluded

\footnotetext{
1 Máster en Docencia Universitaria, Editor Bilingüe de la Universidad de las Regiones Autónomas de la Costa Caribe Nicaragüense-Recinto Universitario Nueva Guinea. Correo: juancarlos.altamirano@uraccan.edu.ni ORCID: https://orcid.org/0000-0003-2244-6014

2 Máster en Docencia Universitaria, Profesora de la Universidad de las Regiones Autónomas de la Costa Caribe Nicaragüense-Recinto Universitario Nueva Guinea. Correo: rubicruzcano@hotmail.com

3 Máster en Género y Desarrollo. Coordinadora del Centro de Estudio e Información de la Mujer Multiétnica de la Universidad de las Regiones Autónomas de la Costa Caribe Nicaragüense-Recinto Universitario Nueva Guinea. Correo: julianafrancis2000@yahoo.com.mx ORCID: https://orcid.org/0000-0002-3384-5443
} 
that the pedagogical capacities of the teachers are very good, that is to say, most of them provide tools for the students to promote their personal development and that of their community, through motivation strategies; however, they do not aim to develop relevant methodologies to facilitate the educational process, especially in intercultural gender relations.

Keywords: Evaluation; performance; teaching; Complementary Courses; Communication; motivation.

\section{Introducción}

La Universidad de las Regiones Autónomas de la Costa Caribe Nicaragüense (URACCAN), enfrenta retos y necesidades relacionados con la calidad, la pertinencia o relevancia de los planes de estudios que se ofrecen a la sociedad, el equilibrio que debe de existir entre las comunidades de aprendizaje, la creación y recreación de conocimientos, saberes y prácticas, y la internacionalización de la Educación Superior en el nuevo contexto globalizado. Es por ello que se propuso en el 2012 que en los planes de estudios, se incorporara la enseñanza del Idioma Inglés como segunda lengua en todos sus currículos, lo que garantizaría no sólo profesionales más competitivos en el mercado laboral, sino también estaría contribuyendo a que el desarrollo de las comunidades de la Costa Caribe de Nicaragua sea más efectivo (URACCAN, 2012), esto exige que los docentes tengan que estar muy preparados especialmente en el proceso de aprender a enseñar y enseñar a aprender a nuestros dicentes un segundo idioma.

Por consiguiente, surge la necesidad de investigar sobre el desempeño de los docentes en los cursos complementarios de Inglés en la URACCAN-Recinto Universitario Nueva Guinea, desde el punto de vista académico de manera que se pueda aportar a una mejor calidad y continuar creciendo en el desarrollo y formación de hombres y mujeres con conocimientos, saberes, capacidades y valores que les harán muy productivos en el contexto de la Costa Caribe y por ende, el país.

\section{Revisión de literatura}

Los docentes pueden transmitir con eficiencia sus conocimientos y llevar a sus estudiantes a la construcción de nuevos conceptos aplicables a la realidad. Es decir, el desempeño docente se define como ejercer docencia de calidad, la que representa un desafío por ser una tarea compleja, altamente exigente y retadora, se hace sumamente necesario que quienes la ejerzan estén debidamente preparados. Una de las más acertadas respuestas a esta exigencia de calidad y excelencia es la constante formación y profesionalización del trabajo docente (Villar, 2004, p. 47). Por consiguiente, esta formación y profesionalización debe ofrecer al docente la oportunidad de adquirir y aplicar nuevos conocimientos para desempeñar su trabajo docente de una forma más eficiente y satisfactoria.

La práctica docente es una actividad profesional que está marcada o delimitada por un marco normativo ya sea nacional o institucional y que debe adecuarse a las realidades. Ser un docente universitario competente desde una concepción humanista de la educación significa, no sólo ser un conocedor de la ciencia que explica, sino también de los contenidos teóricos y metodológicos de la psicología, la pedagogía y la investigación educativa contemporáneas que los capacite para diseñar en sus disciplinas un proceso de enseñanza-aprendizaje potenciador del desarrollo de la personalidad del estudiante (González, 200o).

La URACCAN (2015), se propone desde su misión la formación de hombres y mujeres con conocimientos, saberes, capacidades, valores, principios, actitudes humanistas, sentido del emprendimiento e innovación en equilibrio y armonía con la Madre Tierra para el fortalecimiento de la autonomía de los pueblos. Para la consecución de esta misión, estratégicamente la URACCAN fomenta cursos integrales mediante la formación de competencias comunicativas del Idioma Inglés, la incorporación del Inglés como segunda

lengua en todos sus currículos permite garantizar profesionales más competitivos en el mercado laboral y contribuir al desarrollo de las comunidades de la Costa Caribe de Nicaragua sea más efectivo. 
Esta pertinencia de los procesos de comunicación en la comunidad universitaria, la define Cruz (2014), como la comunicación es un proceso complejo marcado por muchos factores (culturales, cercanía de los interlocutores, estados de ánimo) en el que a pesar de haber diferencias entre las distintas personas existen elementos comunes que son los que permiten que se da el proceso de comunicación. La comunicación requiere que, partiendo de determinados esquemas y experiencias, el emisor represente de manera simbólica aquello que quiere expresar, por otra parte, también el receptor partiendo de sus esquemas y experiencias previas debe codificar e interpretar el mensaje transmitido por el emisor (p. 20). Este autor nos lleva a tomar en cuenta que, en la preparación del estudiantado, se debe de enseñar a ser capaces de expresarse, comunicarse y mantener buenas relaciones humanas, pues la educación y la comunicación son procesos que no pueden estar separados.

Por otra parte, la evaluación del desempeño docente desde la perspectiva de Román y Murillo (2008), tiene el propósito esencial, el análisis y valoración del efecto que produce en los aprendizajes y el desempeño de los estudiantes, el despliegue de las capacidades pedagógicas y socioafectivas de los profesores y profesoras, así como el ambiente relacional que estos profesionales recrean e instalan para la enseñanza y el aprendizaje. Bajo este foco, la calidad del desempeño profesional docente ha de verse reflejado principalmente en los aprendizajes y resultados alcanzados por sus estudiantes (p.4). Además, Escobar (2009) plantea que la evaluación se refiere a evaluar y que es algo más que recoger datos, es un juicio que se va formando de manera continua y cualitativa. Se precisa de una evaluación que permita, además de conocimientos y procedimientos, evaluar modelos de actuación, actitud y valores en coherencia con la concepción ampliada de contenido descrita anteriormente. Se debe tener muy claro cuáles son los conceptos, los procedimientos, valores, competencias, técnicas y las actitudes que deben acompañar a los docentes para evaluar en coherencia con éstos y permitir al mismo docente hacerse una autovaloración de su práctica en el proceso de enseñanza y aprendizaje (p. 52.).

Otro aspecto relevante es la importancia de la motivación en el desempeño docente, de acuerdo con Genovard y Gotzens (1990), las actividades que desarrollan los docentes en función del sistema motivacional en el que se encuentran, los que se denominan un sistema motivacional de la capacidad, un sistema motivacional de responsabilidad moral, un sistema motivacional de dominio de la tarea (p. 3). Los sistemas anteriores nos presentan un tipo de motivación que tratan de asegurar el cumplimiento de en este caso de las acciones de la universidad, la ejecución de las intervenciones educativas en el aula de clases, desde una cultura de colaboración, inclusión, interculturalidad y búsqueda en consenso de las soluciones que cambiarán las situaciones negativas en positivas. De allí, la importancia de la motivación docente, pues se busca atender las diferentes problemáticas que enfrentan en el aula de clases, se ayuda y se evita el malestar, fomentando un clima de comunidad educativa, permitiendo al maestro o futura maestro reconocer la necesidad de no depender de un único elemento, sino de la interacción dinámica de todos los elementos actuantes, que según la filosofía de URACCAN (2012), el proceso de enseñanza y aprendizaje debe estar diseñado para la búsqueda de logros, avances mejores y propósitos para los pilares de la educación integrada en un aprender a conocer, aprender a hacer, aprender a vivir juntos y a aprender a ser.

Se puede finalizar, este apartado teórico, que la motivación puede contribuir a aumentar la competencia profesional y personal, esto significa que las habilidades de escucha, habilidades asertivas, de autocontrol y habilidades de resolución de conflictos, son aprendizajes importantes para los docentes, si se quiere eficacia y calidad en el proceso de enseñanza y aprendizaje, optimizando la satisfacción de los docentes.

\section{Materiales y métodos}

Esta investigación ha evaluado el desempeño de los docentes en los cursos complementarios de Inglés de la Universidad de las Regiones Autónomas de la URACCAN Recinto Universitario Nueva Guinea, durante el primer semestre, 2018. El estudio se sustenta en una metodología con enfoque mixto, avalado por un diseño descriptivo y alcance exploratorio, por ello, se aplicaron instrumentos como guías para la revisión 
documental a materiales impresos y electrónicos, así como cuestionarios con una escala Likert a docentes y estudiantes de la universidad. Todo ello, permitió analizar y valorar los factores que incidieron en el desempeño docente que imparten en los cursos complementarios de Inglés en el proceso educativo. Los participantes en esta investigación han sido 20 docentes que imparten clases en los programas y niveles de los cursos complementarios de inglés, es importante resaltar que 8 son mujeres y 12 hombres. También, se aplicaron a los 5 coordinadores de las áreas académicas: Ciencia de la Educación e Idiomas, Ciencias Económicas, Ciencia, Tecnología y Recursos Naturales y Medio Ambiente, Ciencias de la Construcción y Ciencias de la Salud.

También, se utilizó una codificación para los entrevistados que a continuación se detalla:

\begin{tabular}{ll}
\hline \multicolumn{1}{c}{ Tipo de Entrevistados } & \multicolumn{1}{c}{ Código } \\
\hline \hline Coordinadores Académicos & CA-Informante \\
Docentes & DCT-Informante \\
Estudiantes & EST-Informante \\
\hline
\end{tabular}

\section{Resultados y discusión}

\section{La planificación, organización de los aprendizajes de los cursos complementarios.}

\section{Responsabilidad en la planificación}

La valoración de los coordinadores de áreas y los docentes de los cursos complementarios, se puede constatar esta responsabilidad en la planificación y organización de la asignatura.

De acuerdo a los coordinadores de área, los facilitadores generalmente cumplen con los requisitos adquiridos con la entrega de la planificación de su asignatura, los informes y el cumplimiento del syllabus aunque algunos de ellos no los entregan en tiempo y forma. En este sentido, los coordinadores de área académica expresan que "generalmente el docente cumple con los compromisos adquiridos en el contrato laboral y con la coordinación; sin embargo, algunos de los docentes tienden a ser olvidadizos" (CA-Informante 1). Algunos docentes señalan que el primer día de clases es esencial para la presentación del syllabus a los estudiantes, tal como lo afirma una docente "el primer día de clases le presento a mis estudiantes las unidades, cómo voy a evaluarlos y el tiempo de duración del curso, siempre envío mi syllabus y planes tres días antes del encuentro al coordinador de área" (CA-Informante 2).

\section{Organización de los aprendizajes}

En la organización de los aprendizajes, los docentes establecen y socializan con los estudiantes, las normas y procedimientos para una buena convivencia en el grupo, sin embargo, poco se identifican los objetivos conceptuales, actitudinales y procedimentales, que según el Régimen Académico (URACCAN, 2016) se deben plasmar, enfocados a desarrollar metodologías pertinentes para facilitar el proceso de enseñanza y aprendizaje especialmente en las relaciones con perspectiva intercultural de género y en los contextos interculturales en los que la universidad se desarrolla.

\section{Capacidades pedagógicas en el desarrollo de la asignatura}

Dentro de las capacidades pedagógicas que el profesorado participante del estudio posee, están: fijar la atención de los estudiantes al momento de orientar sus clases presenciales, brindar herramientas al estudiantado para promover su desarrollo personal y el de su comunidad, mediante el uso de medios tecnológicos en la producción de audios y videos cortos, conversaciones que suelen darse en la vida real, para hacer reflexiones y diálogos entre sí. 
Algunos estudiantes expresan que: "nunca ha habido problemas en el salón, ponemos mucha atención y nadie hace relajo" (EST-Informante 1), "necesitamos que el docente sea más dinámico" (EST-Informante 2), "el docente se expresa con seguridad, pero en ocasiones es muy aburrido" (EST-Informante 3), "utiliza dinámicas" (EST-Informante 4), "que la clase sea más práctica, que teórica" (EST-Informante 5), "la docente es dinámica y se expresa con seguridad" (EST-Informante 6), "poco dinámico y es aburrido" (ESTInformante 7), "la clase se considera muy normal, porque no realizamos actividades que nos motiven" (EST-Informante 8). "No, porque se burla de nuestras capacidades" (EST-Informante 9), "Él no da tiempo para las actividades y no le importa si terminamos" (EST-Informante 10), "Durante la clase se comporta de manera arrogante" (EST-Informante 11), "Tienen demasiada petulancia y todo lo hace temporalizado" (EST-Informante 12), "la clase es muy mecánica". (EST-Informante 13)

\section{Cumplimiento y seguimiento con los estudiantes}

Un aspecto importante en la responsabilidad, la planificación y organización de una asignatura, es el cumplimiento y seguimiento con los estudiantes, el que puede valorar por medio de la asistencia puntual y jornada completa de las sesiones de trabajo que incluyen las clases presenciales, clases prácticas, acompañamientos y tutorías, en las que se observó que en las carreras evaluadas, todos los docentes cumplen con su jornada laboral, pero que los docentes especialmente de las carreras de Ingeniería Civil y Licenciatura en Lengua y Literatura Hispánica deben de organizar más clases prácticas fuera del salón del clases.

\section{Proceso de evaluación utilizado en los cursos complementarios}

La evaluación de los aprendizajes es uno de los componentes decisivos del cambio que requerimos en la educación contemporánea. La evaluación debe ser congruente con las definiciones del currículo y es mediante estos procesos que podemos conocer si cada estudiante está construyendo las competencias que le permitan una posición más autónoma y más eficaz en su vida. Según el Modelo Pedagógico de URACCAN (2004), la evaluación de los aprendizajes debe basarse en un modelo que pueda ser aplicado a los diferentes contextos en los que se encuentra la universidad, promoviendo el reconocimiento de la diversidad y las diferencias entre los estilos de aprendizaje, es decir, la evaluación debe ser integral, tomando en cuenta lo endógeno con lo exógeno, para valorar y mejorar nuestro propio contexto. De los resultados obtenidos en esta investigación el $79.22 \%$ de estudiantes opinan que los docentes elaboran las evaluaciones en los cursos complementarios de forma clara y coherente y el 28.78 \% expresan que dichas evaluaciones son poco coherentes. Por otro lado, el $88.89 \%$ de los docentes en estudio expresaron que cumplen coherentemente, dato que coincide con los estudiantes, con sus evaluaciones de una forma clara y en concordancia con los objetivos de la asignatura y la filosofía de URACCAN, Se indagó en los estudiantes en relación al contenido y pertinencia en las evaluaciones obteniendo como resultados que un $64.9 \%$ de los docentes en estudio evalúan a sus estudiantes más allá de conceptos prediseñados y que fomentan en ellos el análisis y la formación del pensamiento crítico. Mientras que un $35.1 \%$ cumple parcialmente. Este dato es un indicador importante que permitirá tanto a docentes, coordinadores de área y autoridades universitarias retomar acciones para mejorar en este aspecto, a través de la puesta en marcha de algunas estrategias para el fortalecimiento de las capacidades docentes.

De acuerdo con la información obtenida por las fuentes primarias de esta investigación los coordinadores de área opinan que en su gran mayoría los docentes promueven la autoevaluación y la coevaluación de sus estudiantes, por su parte los docentes en este estudio afirman que en un $82.7 \%$ cumplen totalmente con este indicador mientras que los demás lo hacen parcialmente, dato que coincide claramente con lo planteado por los estudiantes.

\section{Procesos de comunicación, relaciones interpersonales y trabajo en equipo de los docentes}

\section{- 1. Relaciones interpersonales docente- estudiante}


Tomando como punto de partida la aseveración de Benito (2006) que enuncia que la relación entre profesor- alumno no es solo una fuente de satisfacción del profesor en su trabajo, si no que para el alumno puede constituir una importante fuente de apoyo y motivación y por tanto ejercer cierta influencia en su rendimiento académico los resultados demuestran que un 90\% de los docentes afirmaron que su docente atiende, valora y aclara las dudas planteadas durante el trabajo de la asignatura, un $77 \%$ de los docentes reconoce y corrige sus errores y en un $74 \%$ respeta y valora el conocimiento de los estudiantes. En los hallazgos significativos de este estudio sobresalen comentarios de insatisfacción de estudiantes con algunos docentes, quienes aducen que el $14.3 \%$ de los docentes en el estudio se muestran arrogantes, no corrigen ni aceptan sus errores y no valora ni respeta el conocimiento de los estudiantes.

\section{- 2. Relaciones Interpersonales y trabajo en equipo}

Los coordinadores aducen que un 90\% de los docentes en el estudio interactúan con los diferentes miembros de la comunidad educativa en el marco del respeto y la convivencia armónica para el logro de su trabajo. Manifiestan ser flexibles y respetuosos para aceptar la diversidad de opiniones y críticas constructivas entre sus colegas y comunidad universitaria. Y que además los docentes se muestran comprometidos con la universidad y aportan sugerencias e ideas para el buen desarrollo de proyectos.

\section{- 3. Motivación que incide en la práctica docente de los cursos complementarios de Inglés}

Entre los hallazgos encontrados en este estudio se pudo constatar la valorización de los docentes sobre el nivel de motivación de ellos mismos, qué tanto se sienten motivados al ser docentes URACCAN y qué tanto les motivan sus coordinadores o autoridades de la universidad.

La mayoría de los docentes se sienten muy motivados y los coordinadores expresan ser motivadores de sus docentes. Algunas entre otras razones que mantienen a los docentes con motivación según los mismos son:

- "La URACCAN es única, su modelo pedagógico y su filosofía la hacen muy especial" (DCTInformantes 1...20).

- "El apoyo que brinda a sus docentes con el material que necesitan para sus clases y la facilitación de otros medios a los que podemos acceder todos los docentes" (DCT-Informantes 1...20).

\section{Conclusiones}

En este estudio se ha evaluado el desempeño de los docentes en los cursos complementarios de Inglés de URACCAN, Recinto Universitario Nueva Guinea, el análisis permitió corroborar que la responsabilidad de la mayoría de los docentes en la planificación y organización de la asignatura tiene un buen cumplimiento, los docentes presentan el syllabus en el primer día de clases, las temáticas, los objetivos y la propuesta de evaluación del curso, los planes de práctica en caso que la asignatura lo requiera y negocian las propuestas de evaluación con los estudiantes.

La organización de los aprendizajes en los cursos complementarios no apunta al desarrollo de metodologías pertinentes para facilitar el proceso de la enseñanza y el aprendizaje especialmente en las relaciones interculturales de género. Sin embargo, las capacidades pedagógicas que los docentes poseen son muy buenas, la mayoría brinda herramientas para que el estudiantado promueva su desarrollo personal y el de su comunidad, por medio de estrategias de motivación.

La mayoría del personal docente domina la temática, actualiza los contenidos de la asignatura a la realidad nacional y del mundo, tiene dominio del grupo, atiende las inquietudes de los estudiantes y 
cumple con su jornada laboral, pero algunos docentes deben de organizar clases prácticas fuera del salón de clases, de igual manera necesita retroalimentarse para poder perfeccionar su práctica pedagógica y sus relaciones con los demás miembros de la comunidad, saber si ha sido entendido por sus estudiantes y replantearse sus procedimientos para alcanzar la excelencia universitaria, especialmente en el dominio del idioma inglés.

La mayoría de los docentes de los cursos complementarios evalúan en coherencia con los objetivos de la asignatura, esto quiere decir que las evaluaciones de algunos docentes van dirigidas al desarrollo del pensamiento crítico más allá del concepto y el docente se autoevalúa reconociendo sus errores para mejorar su práctica pedagógica.

La mayoría del estudiantado en estudio exterioriza tener una buena comunicación con su docente, comprenden sus contenidos y consideran agradable la forma en que se imparte la clase. La comunicación y las relaciones interpersonales de los docentes es considerada buena, se involucran en las actividades pedagógicas de la universidad, colaboran activamente e interactúan con los diferentes miembros de comunidad educativa.

Se concluye que los docentes de los cursos complementarios de Idioma Inglés están motivados porque les gusta el modelo pedagógico y la filosofía de la universidad, porque tienen acceso a materiales y medios disponibles, por el apoyo económico para viáticos y alimentación de los docentes que viajan, la buena comunicación y entendimiento entre las diferentes áreas.

\section{Lista de referencias}

Cruz Lablanca, I., (2014). Comunicación efectiva y trabajo en equipo. España. Ministerio de Educación de España.http://site.ebrary.com/lib/bibliouraccansp/reader.action?docID=10914752, ProQuest Ebook Central, https://ebookcentral.proquest.com/lib/bibliouraccansp/detail.action?docID=3224093.

González, V. (2000, abril 17). La Profesionalidad del Docente Universitario desde una Perspectiva Humanista de la Educación. Ponencia presentada en el I Congreso Iberoamericano de Formación de Profesores, Universidad Federal de Santa María, Río Grande del Sur, Brasil. http://www.oei.es/ historico/valores2/gonzalezmaura.htm

Román M. \& Murillo F. Javier (2008). La evaluación del desempeño docente:

Objeto de disputa y fuente de oportunidades en el campo educativo. Revista Iberoamericana de Evaluación Educativa, 10 (5). ISSN-e 1989-0397, Vol. 1, No. 2, 2008. https://dialnet.unirioja.es/ servlet/articulo?codigo $=2789090$

Villar Angulo L, (2004). Programa para la Mejora de la Docencia Universitaria. Madrid, España: Pearson Education.

URACCAN (2016). Proyecto Educativo Institucional (PEI). Managua: URACCAN.

Sureda García, I (2002). Estrategias psicopedagógicas orientadas a la motivación docente: Revisión de un problema. (Spanish). Revista Española de Pedagogía, (No. 221), Pp. 83- 97.

URACCAN (2016). Régimen Académico de la Universidad de las Regiones Autónomas de la Costa Caribe Nicaragüense. Managua: URACCAN. Reforma aprobada en la sesión ordinaria No. 3 del Consejo Universitario de URACCAN efectuada en el Recinto URACCAN Nueva Guinea, del 29 de septiembre al o1 de octubre del 2016. 